\title{
Prevenção À Violência Infantil Utilizando o Programa Act - Raising Safe Kids
}

\author{
Preventing child violence using the ACT- Raising Safe Kids Program
}

Gabriela de Araújo Braz dos Santos*, Ana Cláudia de Azevedo Peixoto, Maria Alice Ribeiro Lins Andrade

Como citar esse artigo. DOS

SANTOS, G.A.B.; PEIXOTO, A.C.A.;

ANDRADE, M.A.R.L.; Prevenção à violência infantil utilizando o programa ACT - Raising Safe Kids. Revista Mosaico, v.12, n.1, p. 76-85, 2021.

Nota da Editora. Os artigos publicados na Revista Mosaico são de responsabilidade de seus autores. As informações neles contidas, bem como as opiniões emitidas, não representam pontos de vista da Universidade de Vassouras ou de suas Revistas.

\begin{abstract}
Resumo
O presente artigo busca demonstrar que ações de prevenção à violência infantil são importantes para a proteção integral desta população. Neste sentido, este artigo tem como objetivo a realização de uma Revisão Integrativa da literatura nacional a respeito dos programas de psicoeducação para cuidadores na prevenção à violência infantil e a utilização do Programa Raising Safe Kids(ACT-RSK). Foi realizado um levantamento bibliográfico nas bases de dados Scielo, BVS, Pepsic e PubMed. Foram encontrados 622 artigos nas plataformas analisadas e, seguindo a leitura dos resumos, foram excluídos os que não se referiam à temática. Somente 9 artigos atenderam aos critérios de inclusão ou exclusão. A literatura aponta que as ações de prevenção à violência infantil, bem como seu combate, são de cunho coletivo, exigindo esforço de todas as esferas que compõem o ambiente da criança. Destaca-se, assim, que as ações preventivas que envolvam diferentes contextos são essenciais para a redução dos riscos de violência, incluindo as estratégias de proteção e promoção da qualidade de vida que integram os princípios fundamentais de atenção e de direitos desta população.
\end{abstract}

Palavras-chave: Programas de Prevenção; Violência infantil; Programa Raising Safe Kids; Educação parental

\section{Introdução}

O reconhecimento do fenômeno da violência contra crianças e adolescentes tem sido um dos grandes problemas da sociedade brasileira, acarretando consequências significativas para esta população. A violência contra crianças é considerada fator de risco, podendo gerar psicopatologias como transtorno do estresse pós-traumático, transtornos de ansiedade, depressão, transtorno de aprendizagem e de personalidade etc. (HABIGZANG; KOLLER, 2011). A exposição à violência nessa fase da vida pode também alterar o funcionamento cognitivo, emocional e comportamental, dificultando o alcance das tarefas evolutivas relacionadas ao desenvolvimento infantil.

É sabido que a violência infantil se tornou um problema de saúde pública e, por isso, deve ser combatida pelo Estado, sociedade, família e agentes sociais que envolvem a criança de uma forma geral (SOUSA et al., 2016). Estudos sobre essa problemática são fundamentais, visto que subsidiam ações de 
cunho preventivo e interventivo, ampliando também a conscientização que envolve essa temática.

Importante ressaltar que as experiências violentas no contexto familiar atuam como fator de vulnerabilidade, diminuindo a proteção necessária ao pleno desenvolvimento da infância (LISBOA; HABIGZANG, 2017). Segundo os casos de abuso sexual analisados pela Instituição de Proteção à infância e adolescência Childhood Brasil (2019), o local apresentado com maior índice de ocorrência da violência foi a casa da vítima (38\%). Levando dados como esse em conta, os programas parentais têm se mostrado como um diferencial nas estratégias preventivas eficazes, uma vez que essas ações são consideradas essenciais para dirimir consequências adversas ao desenvolvimento e bem-estar das crianças (PONTES, 2015).

Refletindo sobre a necessidade de estratégias efetivas de prevenção, entende-se que uma intervenção cujo foco seja direcionado à maneira de agir dos cuidadores passa a ser fundamental, uma vez que pode significar uma alternativa de melhora na qualidade de vida intrafamiliar. Dados da literatura sobre essa temática informam que ensinar crianças maneiras positivas de lidar com as emoções, desenvolvendo habilidades de resolução de conflitos contribui como um fator protetivo ao pleno desenvolvimento da criança (SILVA; WILLIAMS, 2016).

Neste sentido, este artigo tem o objetivo de apresentar o programa ACT-Raising Safe Kids (ACTRSK), como um dos programas indicados para prevenção à violência infantil. O mesmo foi desenvolvido pela American Psychological Association - APA como um programa parental de prevenção universal à violência praticada contra a criança, ao promover atitudes de proteção que sejam contrárias à violência. Dessa forma, proporciona-se um ambiente de relações saudáveis, facilitando os objetivos da prevenção (WOLFE; JAFFE,1999 apud PONTES, 2015).

\section{Teoria Bioecológica e Programas de Psicoeducação}

Considerandoa peculiaridade do desenvolvimento de crianças e adolescentes, pode-se afirmar que esses indivíduos fazem parte dos grupos sociais mais vulneráveis à ocorrência de violência. Dessa maneira, profissionais que estão próximos a este público precisam dar atenção para as especificidades dessas pessoas, buscando alertar para vulnerabilidades, riscos e possibilidades de prevenção, cuidados e proteção (BRASIL, 2010).

Nesse caminho, ao pactuar estratégias preventivas para atenção à saúde de crianças e seus familiares em situação de violência, busca-se a promoção de saúde no sentido de proteção da pessoa e o controle dos possíveis riscos. A priori, o Ministério da Saúde, entende prevenção como "uma estratégia de promoção da saúde, na medida em que previne e controla os agravos, por meio da proteção e defesa de indivíduos e grupos que se encontram em situações de riscos e de vulnerabilidades específicas" (BRASIL, 2010 p. 13).

Ainda de acordo com o Ministério da Saúde (BRASIL, 2010), ações preventivas na comunidade são essenciais para a redução dos riscos de violência. Neste sentido, os objetivos de um programa de prevenção estão voltados ao ensino do uso efetivo das estratégias para mudança dos fatores de risco e a construção do repertório de habilidades sociais e de resolução de problemas, com vistas a sanar as consequências da violência.

Programas de psicoeducação familiar, que visam orientar e ensinar os cuidadores sobre práticas parentais saudáveis, constroem sua base teórica nos conceitos das teorias comportamentalistas e da aprendizagem social (SILVA; WILLIAMS, 2016). Ainda, alguns programas consideram os aspectos cognitivos, se atentando para a influência do vínculo seguro no desenvolvimento saudável (BORTOLINI; WAINER, 2019).

No que se refere à prevenção da violência, determinados programas atuam prevenindo precocemente o ciclo da violência, focando-se no trabalho com adultos importantes na vida da criança e que exerçam influência sobre o seu desenvolvimento (MIGUEL; HOWE, 2006). Neste aspecto, a prevenção precisa alcançar outros contextos além da família. Tal colocação demonstra a necessidade de prevenção do nível micro ao macrossistema para construir mudanças em favorecimento do contexto social. Entende-se por microssistema as relações pessoais, experiências e atividades que a pessoa desenvolve no contexto mais pessoal e íntimo que ela vivencia, e o macrossistema diz respeito ao padrão global relacionado a cultura, ao governo, valores da sociedade, religiões etc., presentes no cotidiano da pessoa.

Em consonância com tal ideia, a Teoria Bioecológicado desenvolvimento,de Urie Bronfenbrenner (1917-2005),pode ser facilmente aplicada em programas de psicoeducação com foco na prevenção à violência, uma vez que prioriza em seus conceitos que crianças influenciam os próprios ambientes onde se encontram quando começam a estabelecer algum tipo de vínculo com outras pessoas e, logo, são influenciadas ao mesmo tempo (BRONFENBRENNER, 1977; 1996).

As características particulares de desenvolvimento de uma pessoa encontram significado e plena expressão na sua interação com um determinado ambiente (MARTINS; SYMANSKI, 2004). Sob o mesmo ponto 
de vista, as características do ambiente onde a criança convive são decisivas para favorecerem ou dificultarem o alcance de seu potencial de desenvolvimento, ou seja, algumas experiências que envolvem a criança ou adolescente, podem deixá-los mais vulneráveis à violência ou podem torná-los mais protegidos, dificultando o acometimento da violência (BRASIL, 2010).

A Teoria Bioecológica compreende os diferentes aspectos da vida, seja o tempo em que o indivíduo vive, os ambientes dos quais participa e as relações que estabelece. Similarmente, os programas de prevenção, cujo objetivo seja a psicoeducação de práticas parentais positivas, buscam avaliar os aspectos de risco dos ambientes e promover relações familiares saudáveis (POLLETO; KOLLER, 2008).

Outrossim, os aspectos do desenvolvimento bioecológico, tais como: o processo-reciprocidade entre as pessoas; a pessoa - características biopsicológicas e as características construídas pelo indivíduo; o contexto - padrão de atividades que envolvem os ambientes em que o indivíduo está inserido; o tempo - influência que a passagem do tempo gera ao longo do ciclo da vida do indivíduo, permitem uma compreensão mais plena do desenvolvimento infantil. Do mesmo modo, programas de prevenção que se propõem olhar os diversos contextos da criança, trabalhar em conjunto com escolas e instituições constroem uma avaliação capaz de identificar as necessidades das crianças relacionadas à situação de violência, oferecendo suporte adequado (PETERSEN, 2011; PONTES, 2015).

De fato, o desenvolvimento humano ocorre permeado por esses processos de interação entre as pessoas, objetos e símbolos, em seu ambiente que são influenciados e influenciam múltiplos elementos que fazem parte de um todo social (MARTINS; SZYMANSKI, 2004). Esses processos são denominados como "processos proximais" entendidos como "formas particulares de interação entre organismo e ambiente, que operam ao longo do tempo e compreendem os primeiros mecanismos que produzem o desenvolvimento humano" (BRONFENBRENNER; MORRIS, 1998, p. 994).

Certamente, ao discutir sobre programas de prevenção à violência, abre-se o debate para a promoção de saúde com o engajamento nas questões sociais. A relação entre os diversos contextos também pode ser considerada na prevenção à violência baseando-se no modelo ecológico para desenvolver fatores de proteção à criança, ajuda às famílias e compartilhamento das habilidades de enfrentamento a violência infantil (PONTES, 2015). Importante ressaltar que o modelo ecológico busca entender a concepção de pessoa na sua passagem pelo desenvolvimento conectado com seu ambiente. Segundo Bronfrenbrenner (1996; p. 18) "a ecologia do desenvolvimento humano envolve o estudo científico de acomodação progressiva mútua, entre um ser humano ativo, em desenvolvimento, e as propriedades mutantes dos ambientes imediatos em que a pessoa em desenvolvimento vive".

A prevenção proporciona desenvolvimento saudável e funciona a partir da conexão entre família e comunidade. Segundo os resultados de um estudo com mães em situação de risco, o abuso infantil diminui quando o conhecimento sobre como educar aumenta e habilidades parentais são reforçadas. Essas habilidades podem ser observadas quando os responsáveis conseguem colocar limites com afeto, quando auxiliam a expressividade emocional dos filhos, ou quando ajudam as crianças na utilização de estratégias funcionais para lidar com as adversidades (DUMONT et al., 2000 apud ANTUNES; MACHADO, 2012). Com isso, programas psicoeducativos estimulam ambiente familiar e rede social favorável a uma promoção de comportamentos adaptativos frente às experiências adversas.

Também, diversos pesquisadores tornam evidente que os cuidadores são figuras estruturantes do desenvolvimento emocional saudável dos seus filhos (BORTOLINI; WAINER, 2019). Assim, o trabalho de prevenção assume grande relevância configurando-se em uma alternativa para trabalhar as consequências da violência infantil, e uma possibilidade de promover mudanças mais profundas nos padrões disfuncionais.

\section{Programa ACT - Educando Crianças em Ambientes Seguros: Uma Revisão Integrativa da Literatura}

O Programa Raising Safe Kids (ACT-RSK), as iniciais ACT significam Adults and Children Together (Adultos e Crianças em Conjunto). O programa ACT baseia-se em uma abordagem psicoeducacional, alinhando aprendizagem de conteúdos teóricos com práticas de habilidades num contexto de interações. O Programa Raising Safe Kids (ACT-RSK), significa Adults and Children Together (Adultos e Crianças em Conjunto) e foi desenvolvido pela American Psychological Association - APA, para atender como um programa parental de prevenção universal à violência praticada contra a criança, e teve uma versão traduzida para o português: Programa ACT para Educar Crianças em Ambientes Seguros (SILVA, 2011). O programa compreende nove sessões e visa o treinamento de profissionais e cuidadores de crianças a definirem comportamentos infantis apropriados a cada etapa do desenvolvimento, enfatizando a importância do papel positivo para a criança no sentido de monitorar e guiar o comportamento desta sem o uso da violência ao utilizar práticas pró-sociais na educação da mesma. Ele também enfatiza o envolvimento de pais nas escolas e comunidades para a prevenção da exposição da criança à violência e foca nove pontos: entrevista 
motivacional e mudança de comportamento; entender os comportamentos das crianças; impacto da exposição da violência na vida das crianças; entender e controlar a raiva (adultos) protetores das crianças; compreender e ajudar crianças quando elas têm raiva; entender e controlar o impacto da mídia eletrônica; estratégias positivas de resolução de conflitos; disciplina para ensinar comportamentos positivos, apropriados; papel dos pais: professores, defensores e cuidadores (SILVA, 2011).

O objetivo deste tópico consiste na realização de uma Revisão Integrativa da literatura sobre o escopo dos programas de psicoeducação para cuidadores na prevenção à violência infantil e a utilização do Programa ACT. Sobre a realização da Revisão Integrativa, as buscas foram realizadas no segundo semestre de 2019 nas bases: Scielo, Pepsic, PubMed e na Biblioteca Virtual em saúde (BVS). Não houve limite para a data de publicação, sendo selecionados artigos, dissertações ou teses, independentemente do tempo de publicação.

Foram realizadas 11 combinações das seguintes palavras-chaves: "ACT: Raising Safe Kids" AND "prevenção à violência AND crianças"; "prevenção à violência" AND "violência contra crianças $e$ adolescentes" AND "ACT"; "programa de prevenção" $A N D$ "violência contra crianças" AND "ACT: Raising safe kids"; "violência contra crianças e adolescentes" AND "praticas parentais" AND "programa ACT"; "violência" AND "crianças e adolescentes"; "violência contra crianças" AND "promoção de saúde e prevenção 'a violência"; "promoção de saúde $e$ prevenção à violência" AND "violência contra crianças e adolescentes"; "práticas parentais" AND "educação parental" AND "violência contra crianças e adolescentes"; "promoção de saúde" AND "promoção dos direitos da criança e do adolescente"; " $A C T$ " $A N D$ "prevenção" AND "violência"; "ACT: Raising safe Kids".

Os critérios de inclusão englobaram: ser um trabalho de prevenção da violência infantil com base na psicoeducação de cuidadores; texto completo e disponível; pesquisas de prevenção à saúde ou propostas de intervenção às crianças e famílias. Para finalizar a pesquisa realizou-se a busca nos títulos e resumos da relação entre os trabalhos selecionados e a temática do presente artigo.

A quantidade de artigos encontrados na primeira busca nos portais foram: "Scielo: 12", "Pepsic: 6", "BVS: 3.154", PubMed: 5". Após a seleção, considerando-se os critérios de inclusão, obteve-se um total de 622 trabalhados. Realizou-se uma segunda busca a partir do assunto e disponibilidade, totalizando, dessa forma, 36 artigos para análise por título e resumo. Após essa etapa foram selecionados 9 estudos paradiscussão.

O quadro 1 apresenta os principais resultados obtidos na revisão, incluindo os objetivos, os instrumentos e os principais resultados dos estudos que foram incluídos na pesquisa. A seguirserá apresentada a discussão destes resultados.

Segundo Silva e Wiliams (2016), o programa ACT tem se mostrado eficaz na prevenção de interações não adaptativas (uso de violência) entre pais e crianças. $\mathrm{O}$ programa se baseou em resultados empíricos de prevenção à violência infantil, incluindo intervenções como: treinamento parental grupal com informações acerca do desenvolvimento da criança, fatores de risco para o desenvolvimento saudável, treino em comunicação e outras habilidades sociais, bem como a resolução de conflitos sem o uso da violência (KNOX et al., 2011 apud SILVA; WILIAMS, 2016). Promover atitudes e comportamentos não violentos reflete uma maneira de se aproximar dos objetivos da prevenção e promoção do desenvolvimento saudável da criança e seus familiares (SILVA; WILLIAMS, 2016).

Para Costa e Brigas (2007), a proteção e a promoção da qualidade de vida de crianças e adolescentes representam desafios, cuja amplitude e complexidade ultrapassam os desafios que as agências de saúde pública habitualmente solucionam. Tais desafios se relacionam com a inabilidade destes indivíduos lidarem sozinhos com as exigências do ambiente, dessa forma, constitui uma população mais vulnerável. Face a esta ideia, vale considerar que crianças e adolescentes são mais vulneráveis à violência, visto tratar-se de seres que exigem do universo adulto a proteção e segurança necessárias para desenvolverem-se melhor (EGRY et al., 2016).

Práticas educativas parentais podem favorecer o desenvolvimento de comportamentos antissociais (resultante de abuso físico, psicológico, negligência e punição inconstante, por exemplo) ou pró-sociais (resultantes de monitoria positiva e comportamento moral), a depender de sua frequência e qualidade (GOMIDE, 2003). Entende-se que as experiências na infância e adolescência são estruturadoras da personalidade. Dessa forma, um ambiente de validação das necessidades emocionais possibilita o desenvolvimento saudável desta personalidade, enquanto um ambiente invalidante pode gerar esquemas disfuncionais (YOUNG et al., 2008). Um ambiente validante compreende a presença de segurança, afeto, apresentação de limites, estímulo a expressão emocional e lazer.

Mussera et al. (2018) discutem que a exposição ao ambiente disfuncional pode gerar vulnerabilidade cognitiva, emocional e física, podendo, dessa forma, estimular o desenvolvimento de sintomas relacionados a diferentes psicopatologias, tais como: ansiedade, depressão, stress, déficit de atenção e doenças psicossomáticas. Nessa sequência, programas parentais 
Quadro 1. Resultado da Revisão Integrativa da Literatura.

\begin{tabular}{|c|c|c|c|c|c|c|}
\hline & AUTOR & ARTIGO & PALAVRA-CHAVE & OBJETIVOS & RECURSOS & AMOSTRA \\
\hline 1 & $\begin{array}{l}\text { M E N E G H E L, } \\
\text { Stela et al. } 2000 .\end{array}$ & $\begin{array}{l}\text { Cotidiano violen- } \\
\text { to: oficinas de pro- } \\
\text { moção em saúde } \\
\text { mental em Porto } \\
\text { Alegre }\end{array}$ & $\begin{array}{l}\text { Violência contra Crianças } \\
\text { e Adolescentes; Promo- } \\
\text { ção da Saúde Mental. }\end{array}$ & $\begin{array}{l}\text { Construir, realizar e avaliar uma oficina } \\
\text { de promoção à saúde com famílias em } \\
\text { situações fragilizadas (Hita,1998) de um } \\
\text { bairro da cidade de Porto Alegre. }\end{array}$ & $\begin{array}{l}\text { Intervenção com oficina de } \\
\text { trabalho }\end{array}$ & $\begin{array}{l}\text { Famílias de baixa ren- } \\
\text { da procedentes do bairro } \\
\text { Partenon em Porto Alegre } \\
\text { - RS }\end{array}$ \\
\hline 2 & $\begin{array}{l}\text { SILVA, Jéssica de } \\
\text { Assis; WILLIA- } \\
\text { MS, Lúcia Caval- } \\
\text { canti de Albuquer- } \\
\text { que. } 2016 .\end{array}$ & $\begin{array}{l}\text { Um estudo de caso } \\
\text { com o programa } \\
\text { parental ACT para } \\
\text { educar crianças } \\
\text { em ambientes se- } \\
\text { guros }\end{array}$ & $\begin{array}{l}\text { Violência contra a crian- } \\
\text { ça, prevenção universal, } \\
\text { programa ACT }\end{array}$ & $\begin{array}{l}\text { Relatar um estudo de caso descrevendo } \\
\text { uma intervenção feita a uma mãe com } \\
\text { o Programa ACT para Educar Crianças } \\
\text { em Ambientes Seguros - programa de } \\
\text { prevenção universal à violência contra a } \\
\text { criança }\end{array}$ & $\begin{array}{l}\text { Questionário de Avaliação do } \\
\text { Programa ACT.Inventário de } \\
\text { Estilos Parentais - IEP (Gomi- } \\
\text { de, 2006) Inventário de Autoa- } \\
\text { valiação paraAdultos de } 18 \text { a } 59 \\
\text { anos (Achenbach\&Rescorla, } \\
\text { 2001) AdultBehavior Check- } \\
\text { List2 (ABCL)ChildBehavior } \\
\text { Checklist3 (CBCL)Diário de } \\
\text { Campo }\end{array}$ & Estudo com uma mãe \\
\hline 3 & $\begin{array}{l}\text { THO MA NIZE, } \\
\text { Angélica Malman; } \\
\text { OLIVEIRA, Bea- } \\
\text { triz Rosana Gon- } \\
\text { çalves; VIEIRA, } \\
\text { Cláudia Silveira. } \\
\text { 2009. }\end{array}$ & $\begin{array}{l}\text { Atenção à crianças } \\
\text { e adolescentes ví- } \\
\text { timas de violência } \\
\text { intrafamiliar por } \\
\text { enfermeiros em } \\
\text { serviços de pron- } \\
\text { to-atendimento }\end{array}$ & $\begin{array}{l}\text { Criança; Adolescente; } \\
\text { Violência doméstica; En- } \\
\text { fermagem }\end{array}$ & $\begin{array}{l}\text { Identificar de que forma acontece o aten- } \\
\text { dimento de enfermagem às crianças e } \\
\text { aos adolescentes vítimas de violência } \\
\text { intrafamiliar nos serviços de pronto- } \\
\text { atendimento de instituições de saúde } \\
\text { conveniadas ao SUS no município de } \\
\text { Cascavel-Paraná. }\end{array}$ & Entrevista semiestruturada & $\begin{array}{l}13 \text { enfermeiros dos servi- } \\
\text { ços de pronto-atendimen- } \\
\text { to de instituições de saú- } \\
\text { de pública integrantes do } \\
\text { Sistema Único de Saúde }\end{array}$ \\
\hline 4 & $\begin{array}{l}\text { MINAYO, Maria } \\
\text { Cecilia de Souza, } \\
\text { et al. } 2018 \text {. }\end{array}$ & $\begin{array}{l}\text { Institucionaliza- } \\
\text { ção do tema da } \\
\text { violência no SUS: } \\
\text { avanços e desafios }\end{array}$ & $\begin{array}{l}\text { Violência; Política de } \\
\text { saúde; Morbidade; Mor- } \\
\text { talidade }\end{array}$ & $\begin{array}{l}\text { Oferecer um panorama das estratégias e } \\
\text { ações implementadas no âmbito do Sis- } \\
\text { tema Único de Saúde, tomando como } \\
\text { norte a Política Nacional de Redução da } \\
\text { Morbimortalidade por Acidentes e Vio- } \\
\text { lência. }\end{array}$ & Análise de dados & Diretrizes da política \\
\hline 5 & $\begin{array}{l}\text { GUIM A R A ES, } \\
\text { Mariana Ramos. } \\
2018 .\end{array}$ & $\begin{array}{l}\text { Criança e adoles- } \\
\text { cente em situação } \\
\text { de violência: o } \\
\text { desafio da atenção } \\
\text { integral e da inter- } \\
\text { setorialidade }\end{array}$ & $\begin{array}{l}\text { Maus-tratos infantis; as- } \\
\text { sistência integral à saúde; } \\
\text { cuidado da criança; ação } \\
\text { intersetorial }\end{array}$ & $\begin{array}{l}\text { conhecer as ações e estratégias de aten- } \\
\text { ção integral à criança e ao adolescente } \\
\text { vítimas de violência no município de } \\
\text { Niterói }\end{array}$ & $\begin{array}{l}\text { Análise de dados, estudo etno- } \\
\text { gráfico, semiestruturada }\end{array}$ & $\begin{array}{l}\text { Fichas de notificação de } \\
\text { violência interpessoal e } \\
\text { profissionais do Projeto } \\
\text { de vigilância às violên- } \\
\text { cias de Niterói }\end{array}$ \\
\hline
\end{tabular}


Quadro 1 (cont.). Resultado da Revisão Integrativa da Literatura.

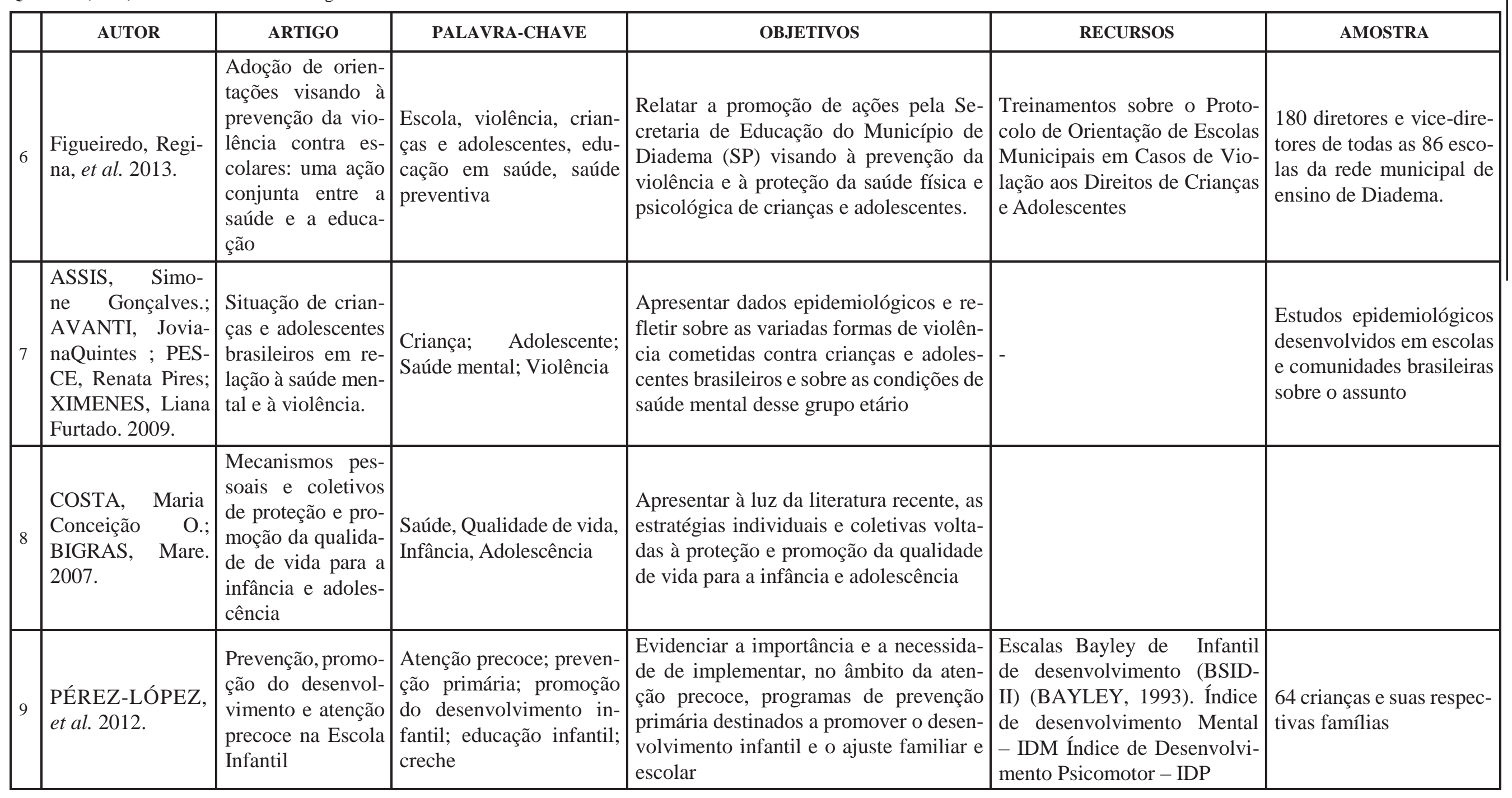


têm se mostrado como estratégias preventivas eficazes à possíveis consequências da violência, bem como, promotores de saúde em diferentes contextos da criança (SILVA; WILIAMS, 2016).

As discussões nos artigos selecionados na Revisão Integrativa corroboram com a ideia de que um ambiente suficientemente bom ao entorno da criança, pode gerar o atendimento das necessidades básicas emocionais, satisfazendo o esforço para proteção, bem como na execução de ações de intervenção contra à violência infantil. Os autores afirmam que as ações de saúde pública voltadas à infância e juventude devem ser dirigidas por adultos capazes de proteger, cuidar e de garantir a eficácia e o impacto da ação-intervenção. Nesta perspectiva, programas psicoeducativos como o ACT contribuem no desenvolvimento de habilidades pró-sociais enfatizando o envolvimento de cuidadores, escolas e comunidades (COSTA; BRIGAS, 2007; SILVA; WILIAMS, 2016).

Umestudo sobre atendimento às crianças vítimas de violência por enfermeiros revelou que profissionais integrantes da equipe de saúde, precisam ter preparo técnico-científico para atuar na prevenção do abuso e negligência, promover a existência e/ou integrar as redes de apoio na comunidade (THOMANIZE; OLIVEIRA; VIEIRA, 2009). A construção de novos conhecimentos e ferramentas melhoram os mecanismos de proteção e cuidados com a saúde e integração social das crianças e adolescentes (COSTA; BRIGAS, 2007).

Diante disso, a família e a escola são sistemas que acompanharão as intervenções com crianças, dado o papel que exercem em suas vidas (NEUFELD et al., 2017).Seguindo esta perspectiva de interação, o estudo de Silva e Wiliams (2016) ilustra que, tal como se pretende em um programa universal de prevenção de violência, o Programa ACT traz importantes benefícios para aprimoramento do estilo parental, contribuindo em conhecimentos e informações novas sobre práticas parentais positivas.

Em consonância com estas ideias, a promoção de saúde voltada à infância e adolescência prioriza a ampla participação das organizações não governamentais - ONG, universidades, governo federal, estadual e municipal, associações de classe, associações comunitárias e comunidades (MINAYO et al., 2018).O trabalho em "rede" se apresenta como uma exigência da realidade social, estimulando o desenvolvimento de práticas integradas, tendo em vista a necessidade de mobilização em torno dos principais problemas sociais que podem comprometer a qualidade de vida, as perspectivas futuras e a integridade de milhares de crianças e adolescentes (COSTA; BRIGAS, 2007; MINAYO et al., 2018).

Em um estudo recente sobre avanços e desafios do tema violência no Sistema Único de Saúde, é possível observar que o estudo da violência contra crianças e adolescentes entrou nas atividades do sistema através dos pediatras, psiquiatras e outros profissionais de saúde e de assistência social que lidavam com esse grupo etário (MINAYO et al., 2018). Segundo as autoras, este movimento ressaltou a atenção para os significativos prejuízos ao crescimento, ao desenvolvimento e à saúde física e mental que a violência proporciona. Florentino (2016) ao citar Romaro e Capitão (2007), destaca que na literatura há concordância entre os especialistas em reconhecer que a criança vítima de abuso e de violência sexual corre o risco de desenvolver uma psicopatologia grave, que perturba sua evolução psicológica, afetiva e sexual.

Nos últimos 40 anos, o tema da prevenção à violência infantil ganhou grande interesse científico e militância, passando a compor a pauta de instituições públicas, privadas e de organizações não governamentais, liderados por profissionais de saúde e movimentos sociais, programas de prevenção primária, secundária(MINAYO et al., 2018). Entretanto, um estudo sobre o desafio da atenção integral às crianças e adolescentes em situação de violência, revela poucas potencialidades na atuação nos casos com ações de prevenção e promoção (GUIMARÃES, 2018).Os resultados desta revisão corroboram com os achados de Guimarães (2018), uma vez que foram poucos os artigos encontrados na pesquisa. Em contrapartida, encontrou-se alguns trabalhos que priorizam a prevenção à violência no Sistema Único de Saúde (SUS). Um destes, foia instituição da Rede Nacional de Prevenção da Violência e Promoção da Saúde e os Núcleos de Prevenção de Violências e Promoção da Saúde em 2004(MINAYO et al., 2018).

Entende-se que as ações em destaque estão a nível nacional e foram coordenadas pela Estratégia de Saúde da Família e pelo Programa Nacional de Segurança Pública na perspectiva de rede de atenção. Uma pesquisa semelhante, visando à prevenção da violência e à proteção da saúde física e psicológica de crianças e adolescentes em um Município em São Paulo, revelou a importância da atuação de secretarias de educação por meio de conhecimentos sobre o fluxo de trabalho e orientações acerca de casos de violência (FIGUEIREDO et al., 2013). Programas de prevenção à violência orientam não somente os pais, mas são extremamente necessários aos profissionais que lidam com este público, uma vez que, ações através de uma prevenção ecossistêmica, agindo através demacro açõesEstado, sociedade, cultura - podem gerar consequências positivas nos microssistemas (famílias, comunidades).

Ao considerar os diversos adultos que participam do ambiente das crianças, promove-se uma intervenção e prevenção na reprodução de padrões sociais de perpetuação de violência (FIGUEIREDO et al., 2013). 
Silva e Wiliams (2016) destacam que o Programa ACT, por meio de seus manuais de aplicação, é um facilitador no entendimento sobre os comportamentos infantis em cada etapa do desenvolvimento para os profissionais e famílias, enfatizando a importância de monitorar e guiar o comportamento sem o uso da violência e prevenir a exposição de crianças à ambientes violentos. Neste sentido, deve-se promover ambientes seguros na expectativa de promoção da saúde e direitos de bemestar e cidadania de crianças e adolescentes, resguardando os seus direitos básicos e contribuindo para que a reprodução de relações de violência cesse na sociedade em geral (FIGUEIREDO et al., 2013; SILVA; WILIAMS, 2016; MINAYO et al., 2018; GUIMARÃES, 2018).

Em uma amostra geral, apenas 1 manuscrito foi encontrado sobre o programa ACT-Raising Safe Kids destacando, então, sua estrutura de encontros e modelos de aplicação. Em sua maioria, os artigos levantados na Revisão Integrativa para estudo final destacam a importância da prevenção à violência nos diferentes sistemas - a família, sociedade e Estado -sem destacar a prática de intervenção a partir de protocolos com encontros sistematizados. Ainda, é possível afirmar que a implementação do Programa ACT em diversos contextos de proteção à criança tem potencial para gerar consequências eficazes no que se refere à prevenção da violência e sua perpetuação, em razão de abordar temas estratégicos sobre desenvolvimento infantil, exposição da criança à violência, resolução de conflitos de maneira positiva e sobre disciplina positiva e estilos parentais (SILVA; WILIAMS, 2016).

Meneghel e colaboradores (2000) realizaram uma pesquisa na Escola de Saúde Pública com pais abusivos incluindo como procedimento o uso de oficinas denominado de "Grupo de Apoio às Famílias do bairro Partenon" e usaram temas como a diminuição dos comportamentos agressivos, história de vida e autoestima. Dessa maneira, os estudos selecionados na revisão integrativa sugerem a implementação de intervenções com os programas como o ACT, bem como, a continuação do trabalho em rede para potencializar o desenvolvimento saudável das famílias e o manejo das consequências da violência (MENEGHEL et al., 2000; SILVA; WILIAMS, 2016; MINAYOet. al, 2018).

Apesar dos avanços das políticas públicas no combate à violência infantil, a implementação de ações que reduzam os casos e as consequências da exposição ainda se apresenta como um grande desafio (GUIMARÃES, 2018). Considerando a violência infantil como problema de saúde pública, os resultados da revisão sobre programas de prevenção à violência infantil mostram-se inexpressivos, se comparados à amplitude deste fenômeno. Fica evidente que muitos desafios ainda persistem, ressaltando que o combate à violência, em suas distintas formas de expressão,precisa tornar-se prioridade nas ações de saúde, educação e assistência, conforme ainda demonstram sua magnitude e impactos na qualidade de vida da população (MINAYO et al., 2018).

\section{Considerações Finais}

Como um problema de saúde pública, entendemos que as ações de prevenção à violência infantil, bem como seu combate, estão entorno de ações coletivas, exigindo esforço de todas as esferas que compõem o ambiente da criança. Discutimos neste artigo a necessidade de ensinar aos cuidadores como educar uma criança em ambiente seguro, bem como, ensiná-los sobre as consequências geradas após exposição à violência no desenvolvimento desse indivíduo. Por fim, as ações preventivas que envolvam diferentes contextos são essenciais para a redução dos riscos de violência, pois ações integradas de proteção são necessidades e direitos fundamentais que geram qualidade no desenvolvimento da infância e da adolescência.

É sabido que crianças se encontram em estágio de desenvolvimento biopsicossocial peculiar e que tem necessidades especiais e imediatas. Assim, o acolhimento das demandas que compreendem os contextos que envolvem a família, escola e grupos sociais se torna uma estratégia de proteção e promoção da qualidade de vida, principalmente, a partir de uma perspectiva ecossistêmica, considerando a importância da interação entre o indivíduo e seu meio ambiente.

\section{Referências}

ANTUNES, Carla; MACHADO, Carla. Abuso sexual na infância e adolescência: Resiliência, competência e coping. Análise Psicológica, vol. 30 n. 1-2, 2012 p. 63-77. Disponível em: http://www.scielo.mec.pt/scielo. php?script=sci_arttext\&pid=S0870-82312012000100007\&lng=pt\&tlng=pt. Acesso em: 13 fev. 2020

ASSIS, Simone Gonçalves.; AVANTI, JovianaQuintes ; PESCE, Renata Pires; XIMENES, Liana Furtado. Situação de crianças e adolescentes brasileiros em relação à saúde mental e à violência.Ciência \& Saúde Coletiva, vol. 14 n. 2, 2009 p. 349-361. Disponível em: https://doi.org/10.1590/S141381232009000200002. Acesso: 14 fev. 2020.

BARRETO, André de Carvalho. Paradigma Sistêmico No Desenvolvimento Humano E Familiar: A Teoria Bioecológica De UrieBronfenbrenner. Psicologia em Revista, v. 22 n. 2, 2016, p. 275-293. Disponível em: https:// dx.doi.org/DOI-10.5752/P.1678-9523.2016V22N2P275. Acesso: $17 \mathrm{fev}$. 2020 .

BORTOLINI, Marcela; WAINER, Ricardo; REIS, Aline Henrique(org). O modelo teórico de orientação a pais na terapia do esquema. In: Terapia do esquema com crianças e adolescentes: do modelo teórico à prática clínica. Campo Grande: Episteme, 2019. p. 193-208.

BRASIL. Lei no 8.069, de 13 de julho de 1990. Dispõe sobre o Estatuto da Criança e do Adolescente e dá outras providências. Diário Oficial [da] República Federativa do Brasil, Brasília, DF, 16 jul. 1990. Disponível em: http://www.planalto.gov.br/ccivil_03/LEIS/L8069.htm\#art266. Acesso: 25 fev. 2020.

BRASIL. Ministério da Saúde (Brasil), Secretaria de Atenção à Saúde, Departamento de Ações Programáticas Estratégicas. Linha de cuidado para 
a atenção integral à saúde de crianças, adolescentes e suas famílias em situação de violências: orientação para gestores e profissionais de saúde. Recurso educacional aberto em português, 2010. Disponível em: https:// portaldeboaspraticas.iff.fiocruz.br/wp-content/uploads/2019/05/linha_ cuidado_criancas_familias_violencias.pdf. Acesso:16 maio 2019.

BRONFENBRENNER, Urie; MORRIS, Pamela A. A ecologia dos processos de desenvolvimento. In.: W. Damon \& RM Lerner (orgs.), Handbook of child psychology: Theoretical models of human development. v. 1, 5 ed., pp. 9931028. New York: John Wiley, 1998.

BRONFENBRENNER, U.A ecologia do desenvolvimento humano: experimentos naturais e planejados. Porto Alegre: Artes Médicas, 1996.

COSTA, Maria ConceiçãoO; BIGRAS, Mare. Mecanismos pessoais e coletivos de proteção e promoção da qualidade de vida para a infância e adolescência. Ciência \& Saúde Coletiva, vol. 12 n. 5, 2007 p. 1101-1109. Disponível em: https://doi.org/10.1590/S1413-81232007000500002. Acesso em: 14 fev. 2020.

EGRY, Emiko Yoshikawa; APOSTOLICO, Maíra Rosa; MORAIS, Teresa Christine Pereira. Enfrentamento da violência infantil numa perspectiva de rede:: o entendimento dos profissionais da atenção primária em saúde. Investigação Qualitativa em Saúde[S.I.], v. 2, p. 1464-1471, 07 jul. 2016. Disponível em: https://proceedings.ciaiq.org/index.php/ciaiq2016/article/ view/904/888 Acesso: 16 fev. 2020.

FLORENTINO, BrunoRicardo B. As possíveis consequências do abuso sexual praticado contra crianças e adolescentes. Fractal: Revista de Psicologia, v. 27, n. 2, p. 139-144, maio-ago. 2015. Disponível em: doi: http://dx.doi.org/10.1590/1984-0292/805.Acesso: 18 jul. 2019

FIGUEIREDO, Reginaet al. Adoção de orientações visando à prevenção da violência contra escolares: uma ação conjunta entre a saúde e a educação. Revista Inst. Saúde, v. 14,n. 3: p. 335-343, ago. 2013. Disponível em: https:// pesquisa.bvsalud.org/portal/resource/pt/biblio-1047123 Acesso em: 17 fev. 2020

GALONI, Luana Luiza; PEIXOTO, Ana Cláudia A. Programa de prevenção e Intervenção contra a violência UFRRJ - LEVICA. Em: Congresso Brasileiro de Terapias Cognitivas da FBTC, 11. Foz do Iguaçu, 2017.

GUIMARÃES, Mariana Ramos. Criança e adolescente em situação de violência: o desafio da atenção integral e da intersetorialidade. Dissertação (Mestrado Acadêmico em Ciências do cuidado em Saúde) - Universidade Federal Fluminense. Niterói, 2018. Disponível em: https://app.uff.br/riuff/ bitstream/1/7121/1/Mariana\%20Ramos\%20Guimaraes.pdf. Acesso: 19 jul. 2019

HABIGZANG, Luísa Fernanda; KOLLER, Silvia Helena. Terapia cognitivo comportamental para crianças e adolescentes vítimas de abuso sexual. In: PETTERSEN, Circe Salcides; WAINER, Ricardo (Orgs). Terapias cognitivocomportamentais para crianças e adolescentes: ciência e arte. Porto Alegre: Artmed, 2011. p. 299-310.

KEITA, Gwen Puryear.Something to celebrate.Monitor on Psychology, [S.I.], v. 42, n. 5, p. 45., 2011.Disponível em: https://www.apa.org/monitor/2011/05/ itpi. Acesso: 19 jul. 2019

LISBOA, Carolina Saraiva de Macedo; HABIGZANG, Luísa F. Violência contra adolescentes.In: NEUFELD, Carmen Beatriz (Org). Terapia Cognitivocomportamental para adolescentes: uma perspectiva transdiagnóstica e desenvolvimental. Porto Alegre, Artmed, 2017. p. 400.

MARTINS, Edna; SZYMANSKI, Heloisa (2004). A abordagem ecológica de UrieBronfenbrenner em estudos com famílias. Estudos e Pesquisas em Psicologia, UERJ, RJ, a. 4,n. 1, 2004,p. 63-77, $1^{\circ}$ Semestre. Disponível em: https://www.e-publicacoes.uerj.br/index.php/revispsi/article/ view/11111/8805 Acesso em: 22 Jul. 2019

MENEGHEL, Stela; ARMANI, Teresa; SEVERINO, Rosa; GARCIA, Ana Maria; MAFIOLETI, Beno; FOCHI, Eunice; RODRIGUES, Fabiana; ARMANI, Luciara; OLIVEIRA, Mara; RODRIGUES, Rosiclair. Cotidiano violento: oficinas de promoção em saúde mental em Porto Alegre.Ciência \& Saúde Coletiva, v. 5, n. 1, 2000 p. 193-203. Disponível em: https://doi. org/10.1590/S1413-81232000000100017.Acesso: 18 jul. 2019.

MIGUEL, Jessica J.;HOWE, Tasha R. Implementing \& evaluating a national early violence prevention program at the local level: Lessons from ACT (Adults \& Children Together) Against Violence. Journal of Early Childhood \& Infant Psychology, v. 2, 17- 38, 2006.Disponívelem: https://www. researchgate.net/publication/309105673_Implementing_and_evaluating_a_ national early violence prevention program at the local level Lessons from_AC̄T_Adults_and_Children_Together_Against_Violence. Acesso: 15 maio 2019 .

MINAYO, Maria Cecilia de Souza, ASSIS, Simone Gonçalves, (org.). Novas e velhas faces da violência no século XXI: visão da literatura brasileira do campo da saúde. Rio de Janeiro: Editora Fiocruz; 2017.

MINAYO, Maria Cecilia de Souza; SOUZA, Edinilsa Ramos de; SILVA, Marta Maria Alves da; ASSIS, Simone Gonçalves de. Institucionalização do tema da violência no SUS: avanços e desafios. Ciência \& Saúde Coletiva, v. 23,n.6, 2018. Disponível em: https://doi.org/10.1590/141381232018236.04962018. Acesso:18 jul.2019

MUSSERA, Nicole; ZALEWSKIA, Maureen; STEPPB, Stephanie; LEWISA, Jenifer.A systematic review of negative parenting practices predicting borderline personality disorder:are we measuring biosocial theory's 'invalidating environment'? Clinical Psycholology Review, v. 6, p 1-16, 2018. Disponível: https://doi.org/10.1016/j.cpr.2018.06.003. Acesso: 19 jul. 2019.

NEUFELD, Carmen Beatriz (org.). Terapia Cognitivo-comportamental para adolescentes: uma perspectiva transdiagnóstica e desenvolvimental. Porto Alegre, Artmed, 2017. 400 p.

PÉREZ-LÓPEZ, Julio; MARTÍNEZ-FUENTES, María Teresa; DÍAZHERRERO, Ángela; BRITO DE LA NUEZ, Alfredo G. Prevención, promocióndeldesarrollo y atencióntempranaenlaEscuela Infantil. Educar em Revista, n. 43, 2012, p. 17-32. Disponível em: https://doi.org/10.1590/ S0104-40602012000100003. Acesso: 18 jul. 2019

PETERSEN, Circe Salcides. Avaliação inicial de crianças: a dimensão bioecológica do desenvolvimento humano. In: PETERSEN, Circe Salcides; WAINER, Ricardo. Terapias cognitivo-comportamentais para crianças e adolescentes: ciência e arte- Porto Alegre: Artmed, 2011.p.32-45.

POLETTO, Michele; KOLLER, Silvia Helena. Contextos ecológicos: promotores de resiliência, fatores de risco e de proteção. Estudos de Psicologia, Campinas, v. 25, n. 3., 2008 p. 405-416. Disponível em: https:// www.scielo.br/scielo.php?pid=s0103-166x2008000300009\&script=sci abstract\&tlng=pt . Acesso: 19 jul. 2019.

PONTES, Luciana Barbalho. Avaliação da eficácia de programa de capacitação parental universal (ACT) pelo uso da observação. Dissertação (Mestrado) do Programa de Pós-Graduação em Psicologia. Universidade Federal de São Carlos, São Paulo, 2015. Disponível em: https://repositorio. ufscar.br/handle/ufscar/7215. Acesso: 18 de jul. 2019

RIOS, Karyne de Souza Augusto.; WILLIAMS, Lúcia Cavalcanti de Albuquerque. Intervenção com famílias como estratégia de prevenção de problemas de comportamento em crianças: uma revisão. Psicologia em Estudo, [S.L.], v. 13, n. 4, p. 799-806, dez. 2008. FapUNIFESP (SciELO). Disponível em: https://www.scielo.br/scielo.php?script=sci_arttext\&pid=S1413-737220 08000400018\&lng=pt\&nrm=iso\&tlng=pt. Acesso: 20 fev. 2020.

SILVA, Julia.Programa ACT para Educar Crianças em ambientes seguros. American Psychological Association. Washington, 2011.

SILVA, Jéssica A.; WILLIAMS, Lúcia Cavalcanti A. Um Estudo de caso com o programa parental ACT para educar crianças em ambientes seguros. Temas em Psicologia, [s.1.], v. 24, n. 2, p. 743-755, 2016. Associação Brasileira de Psicologia. Disponível em: https://dx.doi.org/10.9788/TP2016.2-19Pt. Acesso: 21 fev. 2020.

SOUSA, Grazielly Mendes; DAMASCENO, KamillaChrystinaFerreira; BORGES, Lívia.deCarvalhoFarias. Estratificação dos tipos de violência notificados pelo SINAN, no município de Porto Nacional, TO, em 2014 Revista Interface (Porto Nacional), [S.1.], n. 11, jun. 2016. ISSN 2448-2064 2016. Disponível em: https://sistemas.uft.edu.br/periodicos/index.php/ interface/article/view/2134.Acesso: 23 set. 2019.

THOMANIZE, Angélica Malman; OLIVEIRA, Beatriz Rosana Gonçalves VIEIRA, CláudiaSilveira. Atenção a crianças e adolescentes vítimas de violência intrafamiliar por enfermeiros em serviços de pronto-atendimento. Revista eletrônica de enfermagem, v. 11, n.4. 2009, p.830-40. Disponível em: http://www.fen.ufg.br/revista/v11/n4/v11n4a08.htm. Acesso: 15 ago. 2019

WOLFE, David A.; JAFFE, Peter G. Emerging strategies in the prevention of domestic violence. Futuresof Children, v. 9, n. 3, 1999, p. 133-144. Disponível em: https://www.jstor.org/stable/1602787?origin=crossref\&seq= 1. Acesso: 17 de jul. de 2019 
dos Santos et al., 2021.

YOUNG, Jeffrey; KLOSKO, Janet; WEISHAAR, Marjorie. Terapia do

esquema: Guia de técnicas Cognitivo-comportamentais inovadoras. Porto

Alegre: Artmed, 2008. 